CIENCIAMATRIA

Revista Interdisciplinaria de Humanidades, Educación, Ciencia y Tecnología

Año VI. Vol. VI. N¹. Edición Especial. 2020

Hecho el depósito de ley: pp201602FA4721

ISSN-L: 2542-3029; ISSN: 2610-802X

Universidad Nacional Experimental Francisco de Miranda (UNEFM). Santa Ana de Coro. Venezuela

Marcia Lorena Cabrera-Abad; Darwin Gabriel García-Herrera; María Isabel Álvarez-Lozano; Juan Carlos Erazo-Álvarez

\title{
DOI 10.35381/cm.v6i1.334
}

Trabajo colaborativo en el aprendizaje y relación social de los estudiantes con necesidades educativas especiales

\section{Collaborative work in the learning and social relationship of students with special educational needs}

\author{
Marcia Lorena Cabrera-Abad \\ marcia.cabrera@psg.ucacue.edu.ec \\ Universidad Católica de Cuenca, Azogues \\ Ecuador \\ https://orcid.org/0000-0002-2814-4722 \\ Darwin Gabriel García-Herrera \\ dggarciah@ucacue.edu.ec \\ Universidad Católica de Cuenca, Azogues \\ Ecuador \\ https://orcid.org/0000-0001-6813-8100 \\ María Isabel Álvarez-Lozano \\ mialvarezl@ucacue.edu.ec \\ Universidad Católica de Cuenca, Azogues \\ Ecuador \\ https://orcid.org/0000-0001-8029-1933 \\ Juan Carlos Erazo-Álvarez \\ jcerazo@ucacue.edu.ec \\ Universidad Católica de Cuenca, Cuenca \\ Ecuador \\ https://orcid.org/0000-0001-6480-2270
}

Recibido: 25 de abril de 2020

Aprobado: 10 de junio de 2020 


\title{
RESUMEN
}

La investigación tuvo como objetivo analizar el trabajo colaborativo en el aprendizaje y la relación social de los estudiantes con necesidades educativas especiales. Se abordó un enfoque mixto, descriptivo correlacional. Los resultados evidencian un conocimiento del aprendizaje colaborativo pero poca inclusión educativa de niños con necesidades educativas especiales, información clave para establecer una propuesta que cumpla con los requisitos de una verdadera inclusión educativa, por lo que se pudo encontrar que los docentes están conscientes que dentro de sus aulas existen educandos que requieren mayor atención a sus necesidades, sin embargo se debe reforzar el conocimiento sobre inclusión educativa y las metodologías para garantizar la tan anhelada educación de calidad, por otro lado el profesorado aplica el trabajo colaborativo en el aula, el inconveniente es que generalmente no se siguen los pasos que el mismo establece, para que todos y cada uno de los integrantes participen activamente en el grupo.

Descriptores: Educación especial; aprendizaje activo; autoaprendizaje; experimento educacional. (Palabras tomadas del Tesauro UNESCO).

\begin{abstract}
The research aimed to analyze collaborative work in learning and the social relationship of students with special educational needs. A mixed, descriptive correlational approach was addressed. The results show a knowledge of collaborative learning but little educational inclusion of children with special educational needs, key information to establish a proposal that meets the requirements of true educational inclusion, so it could be found that teachers are aware that within In their classrooms there are students who require greater attention to their needs, however, knowledge on educational inclusion and methodologies must be reinforced to guarantee the long-awaited quality education, on the other hand, teachers apply collaborative work in the classroom, the inconvenience is that the steps that it establishes are generally not followed, so that each and every one of the members actively participates in the group.
\end{abstract}

Descriptors: Special needs education; activity learning; self instruction; educational experiments. (Words taken from the UNESCO Thesaurus). 


\section{CIENCIAMATRIA}

Revista Interdisciplinaria de Humanidades, Educación, Ciencia y Tecnología

Año VI. Vol. VI. N¹. Edición Especial. 2020

Hecho el depósito de ley: pp201602FA4721

ISSN-L: 2542-3029; ISSN: 2610-802X

Universidad Nacional Experimental Francisco de Miranda (UNEFM). Santa Ana de Coro. Venezuela

Marcia Lorena Cabrera-Abad; Darwin Gabriel García-Herrera; María Isabel Álvarez-Lozano; Juan Carlos Erazo-Álvarez

\section{INTRODUCCIÓN}

Hablar de inclusión educativa relacionada con las estrategias metodológicas sin duda constituye un gran reto, por cuanto es un tema que requiere reforzarse para que sea mejor utilizado en beneficio de los estudiantes de nuestra zona y porque no de nuestro país, por lo que mediante este trabajo investigativo se pretende dar mejores herramientas para este fin, dirigido a toda la comunidad educativa, esto es a estudiantes, docentes y representantes legales; con especial énfasis en esta ocasión a los docentes.

De acuerdo con (Opertti, 2009), adscrito a la UNESCO quien menciona que en varias reuniones co-organizadas por la Oficina Internacional de Educación (OIE) de la UNESCO se han planteado los temas de inclusión educativa, donde se refiere que el principal inconveniente es pasar de la integración a la inclusión, para esto se debe generar nuevas prácticas inclusivas en las distintas instituciones del mundo, asumiendo un enfoque que implemente las condiciones necesarias para pasar a ser una sociedad inclusiva, tomando en cuenta que es un desafío perentorio sobre todo para el futuro de la sociedad.

Considerando que de acuerdo con (Domínguez, López, Pino, \& Vázquez, 2015), la inclusión es aquella que permite la modificación de los aspectos más importantes del ámbito educativo como: la estructura, el funcionamiento y las propuestas pedagógicas, con la finalidad de que todos los estudiantes adquieran un aprendizaje significativo, en igualdad de condiciones. En este sentido, la inclusión va más allá de estandarizar las Instituciones Educativas, con estudiantes de necesidades educativas especiales dentro de los establecimientos públicos y privados, ya que busca no solo incluirlos físicamente sino también hacer una reforma totalitaria del plan de estudios con el que se maneja la institución.

Se debe tener en cuenta el aporte de (Ramírez, 2017) en su estudio, realizado en Colombia donde comenta que de acuerdo con los resultados obtenidos la inclusión debe ser vista como una oportunidad de los niños, niñas y jóvenes para ser más libres, motivados a alcanzar sus metas. En cuanto a la percepción del docente, manifiesta que el mismo está siempre buscando diferentes estrategias de incluir a todos los estudiantes en su proceso de diferentes formas, sin embargo, los esfuerzos no han rendido los frutos 


\section{CIENCIAMATRIA}

Revista Interdisciplinaria de Humanidades, Educación, Ciencia y Tecnología

Año VI. Vol. VI. N¹. Edición Especial. 2020

Hecho el depósito de ley: pp201602FA4721

ISSN-L: 2542-3029; ISSN: 2610-802X

Universidad Nacional Experimental Francisco de Miranda (UNEFM). Santa Ana de Coro. Venezuela

Marcia Lorena Cabrera-Abad; Darwin Gabriel García-Herrera; María Isabel Álvarez-Lozano; Juan Carlos Erazo-Álvarez

esperados; en cambio en el caso de la familia, todavía no está consciente de las necesidades de estos niños ya que solo han dejado este trabajo en manos del docente. Por otro lado, el trabajo colaborativo como metodología activa que aporta a la inclusión de acuerdo con (González-Vargas, 2014) manifiesta que "el trabajo colaborativo en la escuela primaria constituye un proceso de aprendizaje interactivo que conjuga esfuerzos e invita a participar y trabajar en equipo" (p.116). Con este aporte es significativo indicar que sin duda este tipo de metodología podría mejorar no solo la forma de aprendizaje y desarrollo del niño de inclusión, sino también la relación con los demás estudiantes, debido a la interacción que este, demanda.

Si bien el estado a través del gobierno ha establecido políticas para incluir a dichos estudiantes, el resultado no ha sido precisamente el esperado, por lo que es importante buscar nuevas formas que admitan una verdadera inclusión educativa. Entonces conociendo que este problema no solo afecta a estudiantes sino también a docentes, a menudo no cuentan con los recursos metodológicos y didácticos necesarios para atender los diferentes tipos y grados de necesidad educativa, otro aspecto a resaltar es también la inversión de tiempo que requiere hacer el docente en este tipo de estudiantes, tiempo que por lo general no es suficiente, lo cual hace que se descuide a los demás estudiantes. Por lo tanto, es necesario poner en práctica una de las metodologías activas más eficientes, con el objetivo de analizar su incidencia y proponer el trabajo colaborativo para generar escenarios de inclusión educativa.

\section{Referencial teórico}

Según un estudio realizado en España por (Fernández, 2013) sobre la inclusión en los centros educativos del mencionado país, encuentra que el compromiso con la inclusión social dentro de los centros educativos contribuye a la motivación y el éxito escolar, con la aplicación de metodologías activas con la finalidad de lograr un aprendizaje significativo en todos los estudiantes. Quienes desarrollan competencias gracias a la innovación y la creatividad del educando. Con este estudio se puede determinar que el 


\section{CIENCIAMATRIA}

Revista Interdisciplinaria de Humanidades, Educación, Ciencia y Tecnología

Año VI. Vol. VI. №1. Edición Especial. 2020

Hecho el depósito de ley: pp201602FA4721

ISSN-L: 2542-3029; ISSN: 2610-802X

Universidad Nacional Experimental Francisco de Miranda (UNEFM). Santa Ana de Coro. Venezuela

Marcia Lorena Cabrera-Abad; Darwin Gabriel García-Herrera; María Isabel Álvarez-Lozano; Juan Carlos Erazo-Álvarez

docente es una pieza fundamental en este proceso, ya que es el que guía y motiva la ejecución de proyectos sin discriminación alguna (Arroyo-Carrera, et al., 2020).

Por otro lado, el estudio de (Álvarez, Grau, \& Pertegal, 2016) realizado en Venezuela, coincide en cierta manera con el de Fernández, en los resultados se ha encontrado que el trabajo colaborativo como metodología para la inclusión ha podido resolver los trabajos propuestos de forma eficaz y práctica de una forma participativa, con algunas dificultades de adaptación por tratarse de una nueva metodología o mejor dicho una metodología bien aplicada, pese a dichas dificultades se observó un grado de satisfacción alto en el estudiantado, sobre todo por la familiarización con los materiales utilizados para la aplicación de la metodología, por la mejora de la comprensión y el grado de productividad obtenido. Esto quiere decir que de acuerdo con los estudios realizados en 2 países diferentes las metodologías activas han sido una excelente herramienta para la relación social de los estudiantes y para la adquisición adecuada de los contenidos propuestos por el docente (Argandoña-Mendoza, et al., 2020).

Como menciona (Vega, 2005) las Necesidades Educativas Especiales [NEE] hacen referencia a las dificultades concretas o generales para aprender o adquirir conocimientos, de las asignaturas de la malla curricular, para lo que se debe emplear más o diferentes recursos, con el objetivo de que el estudiante cumpla con los propósitos educativos planteados por el docente; además se debe tener en cuenta que los estudiantes de NEE no solo son aquellos que tienen alguna discapacidad, sino también aquellos que puedan enfrentar situaciones que no les permitan desenvolverse de la misma forma que los demás estudiantes, en relación a los aprendizajes necesarios para cursar un grado o curso y necesita de ayudas y recursos diferentes, de forma temporal o permanente (García-Cedeño, et al., 2020)

De acuerdo con la autora los estudiantes con necesidades educativas especiales, no deben ser reconocidos solo por alguna discapacidad física o intelectual, sino también debe ser analizado su contexto educativo, familiar o social, que permitirán determinar las razones por las que no puede aprender o por las que necesita más tiempo y recursos 


\section{CIENCIAMATRIA}

Revista Interdisciplinaria de Humanidades, Educación, Ciencia y Tecnología

Año VI. Vol. VI. N¹. Edición Especial. 2020

Hecho el depósito de ley: pp201602FA4721

ISSN-L: 2542-3029; ISSN: 2610-802X

Universidad Nacional Experimental Francisco de Miranda (UNEFM). Santa Ana de Coro. Venezuela

Marcia Lorena Cabrera-Abad; Darwin Gabriel García-Herrera; María Isabel Álvarez-Lozano; Juan Carlos Erazo-Álvarez

para la adquisición de los contenidos establecidos por el docente (Saltos-Cedeño, et al., 2020)

Por otro lado (Martín, González, Navarro \& Latingua, 2017) manifiestan que el hecho de que el sistema educativo permita el acceso a las instituciones educativas regulares, no lo es todo para la inclusión educativa, que sin duda es un gran paso, pero también es necesario crear escenarios adecuados para la igualdad de oportunidades en todos los niveles y consentir que por medio de diferentes metodologías como pueden ser las activas, ellos tengan el éxito deseado en su aprendizaje. Por lo que se ha mencionado se podría decir que las metodologías activas deben ir de la mano con otros factores del contexto escolar, sin embargo, es determinante para que los estudiantes de inclusión tengan la oportunidad de poner en práctica sus habilidades y explotar al máximo sus capacidades (Saltos-Cedeño, et al., 2020).

Así mismo, (Álvarez, Grau, \& Pertegal, 2016), hacen un aporte sobre el trabajo colaborativo y mencionan que este permite dejar atrás las clases tradicionales, para que el estudiante se convierta en el artífice de la adquisición de los aprendizajes, además, intenta dar opciones a los educandos de estrategias que estimulen la participación activa de todos dentro del aula. De acuerdo a este aporte, el trabajo colaborativo busca darle protagonismo al estudiantado, para alcanzar sus propósitos en el aprendizaje, cada persona dentro del grupo es una pieza fundamental para el desarrollo correcto del trabajo encomendado, por lo que la participación de los estudiantes de inclusión sería indispensable, asimismo con la interacción que requiere, la relación con sus pares, podría mejorar significativamente (Quevedo-Álava, et al., 2020).

Además (Echeita \& Duk, 2008) mencionan que el primer paso para la inclusión es el acceso de todos los niños, niñas y jóvenes sin exclusión alguna, tengan admisión a los centros de estudio regular, y el segundo paso sería que se incluyan a una educación de calidad e igualdad de oportunidades; sensibilizando a los demás la importancia de tener una buena relación, para mejorar la vida estudiantil de cada uno de los educandos. No se puede hablar de una inclusión cuando no se ha trabajado antes con los demás estudiantes sobre todo en el ámbito del respeto a las diferencias de los demás, es por 


\section{CIENCIAMATRIA}

Revista Interdisciplinaria de Humanidades, Educación, Ciencia y Tecnología

Año VI. Vol. VI. N¹. Edición Especial. 2020

Hecho el depósito de ley: pp201602FA4721

ISSN-L: 2542-3029; ISSN: 2610-802X

Universidad Nacional Experimental Francisco de Miranda (UNEFM). Santa Ana de Coro. Venezuela

Marcia Lorena Cabrera-Abad; Darwin Gabriel García-Herrera; María Isabel Álvarez-Lozano; Juan Carlos Erazo-Álvarez

eso que la calidad de vida de los estudiantes dentro de los centros educativos fiscales se ha visto afectada, sobre todo de aquellos que tienen alguna dificultad de aprendizaje o una discapacidad (Vélez-Loor, et al., 2020).

Por otro lado, (Aguilar, 2013) manifiesta que la atención a la diversidad como función del Estado intenta conseguir un involucramiento social, en defensa de algunos valores teniendo como base la educación de la mayoría de la población; estos cambios y la aceptación de la sociedad de la inclusión dependería de la modernización y desarrollo de la misma. Entonces está claro que para que exista una verdadera inclusión se debe empezar por transformar la sociedad y el trabajo colaborativo puede permitir que una parte de esa sociedad que conforma el ámbito educativo empiece por ese cambio, por la participación activa de todos los miembros del grupo (Peche-Cruz \& Giraldo-Supo, 2019).

\section{MÉTODO}

Para esta investigación se recurrió a un enfoque mixto con alcance descriptivo correlacional no experimental transversal, de acuerdo con (Dabdub-Moreira \& PinedaCordero, 2015) el enfoque mixto permite comprender de mejor manera los estudios realizados en humanos y su interacción social, permitiendo a los investigadores incorporar datos desde numéricos hasta verbalizaciones de los actores del universo 0 muestra que se plantea (Ávila-Sacoto, Erazo-Álvarez, Narváez-Zurita, \& Erazo-Álvarez, 2019).

En este caso es relevante debido a que el enfoque nos permitió analizar cuáles son los niveles de conocimiento de los docentes de la Unidad Educativa Los Cañaris del cantón Azogues, con respecto a la inclusión educativa y como incide el trabajo colaborativo en su práctica con los estudiantes en cuestión. Se acudió al uso de encuestas desde los aportes de (Sarduy, 2007), describiendo la encuesta como un tipo de investigación cuantitativa, que permite obtener datos numéricos sobre determinadas variables indispensables para el desarrollo de la investigación y posteriormente en los resultados se verá reflejada la realidad del universo con el que se trabajó. 


\section{CIENCIAMATRIA}

Revista Interdisciplinaria de Humanidades, Educación, Ciencia y Tecnología

Año VI. Vol. VI. N¹. Edición Especial. 2020

Hecho el depósito de ley: pp201602FA4721

ISSN-L: 2542-3029; ISSN: 2610-802X

Universidad Nacional Experimental Francisco de Miranda (UNEFM). Santa Ana de Coro. Venezuela

Marcia Lorena Cabrera-Abad; Darwin Gabriel García-Herrera; María Isabel Álvarez-Lozano; Juan Carlos Erazo-Álvarez

En este sentido al querer analizar el nivel de conocimientos de los docentes sobre el tema en cuestión, es importante obtener datos numéricos donde se interpretaron los resultados de acuerdo a las variables planteadas, por medio de la técnica del chi-cuadrado y el análisis individual de tablas que contienen variables indispensables para la obtención de la información necesaria (Calle-Abril, Erazo-Álvarez, \& Narváez-Zurita, 2020).

Por otro lado, para el enfoque cualitativo se realizó un grupo focal que de acuerdo con (Hamui-Sutton \& Varela-Ruiz, 2013) es aquel que ayuda a entender el sentir y pensar de las personas, además explorar sus experiencias interactuando con los demás miembros del grupo para entender las razones de sus criterios, obteniendo una gama de discernimientos.

La encuesta se realizó a 35 docentes de la Unidad Educativa Los Cañaris de la ciudad de Azogues, provincia del Cañar, Ecuador, con un formulario de 8 preguntas, validado por medio de Alfa de Cronbach con 0,717, en el lapso de una semana incluyendo la obtención de los resultados y posteriormente se realizó el grupo focal con 4 maestros de la misma institución (Argudo-Tello, Erazo-Álvarez, \& Narváez-Zurita, 2019).

Para justificar el trabajo investigativo se planificó el desarrollo de 2 instrumentos como son la encuesta y el grupo focal, mismos que tienen por objeto visualizar la realidad fáctica de la inclusión educativa en la Unidad Educativa Los Cañaris. La encuesta realizada estuvo dirigida a 35 docentes del establecimiento antes referido, con la finalidad de determinar el nivel de conocimiento sobre inclusión educativa y metodologías activas así como su aplicabilidad en la práctica diaria de la actividad de los encuestados, para lo cual se ha realizado un formulario de 8 preguntas tendientes a obtener respuestas, sobre los problemas en análisis.

Se incluyó tambien el desarrollo del grupo focal con la finalidad de afianzar las respuestas obtenidas en la encuesta, pero esta vez dirijido a un grupo de 4 docentes seleccionados por cada nivel educativo con la inclusión de tres temas enfocados a través de una reunion presencial, utilizando medios telemáticos, debido al estado de excepeción que rige en el país, para culminar con preguntas dirigidas a obtener información sobre inclusión educativa, estrategias que se manejan con niños de NEE y el trabajo colaborativo. Los 
CIENCIAMATRIA

Revista Interdisciplinaria de Humanidades, Educación, Ciencia y Tecnología

Año VI. Vol. VI. N¹. Edición Especial. 2020

Hecho el depósito de ley: pp201602FA4721

ISSN-L: 2542-3029; ISSN: 2610-802X

Universidad Nacional Experimental Francisco de Miranda (UNEFM). Santa Ana de Coro. Venezuela

Marcia Lorena Cabrera-Abad; Darwin Gabriel García-Herrera; María Isabel Álvarez-Lozano; Juan Carlos Erazo-Álvarez

resultados de estos dos instrumentos hacen parte del presente trabajo conforme se detallan y explican a continuación.

\section{RESULTADOS}

\section{Tabla 1}

Tabla de contingencia Uso de trabajo colaborativo * Inclusión educativa es atender las necesidades de todos y cada uno de los estudiantes sin distinción o discriminación.

Inclusión educativa es atender las necesidades de todos y cada uno de los estudiantes sin distinción o discriminación.

\begin{tabular}{cccccc} 
& $\begin{array}{c}\text { Algo en } \\
\text { desacuerdo }\end{array}$ & $\begin{array}{c}\text { Algo de } \\
\text { acuerdo }\end{array}$ & $\begin{array}{c}\text { Completamente } \\
\text { de acuerdo }\end{array}$ & Total \\
\hline Uso de trabajo colaborativo & No aplica & 1 & 4 & 22 & 27 \\
Total & Aplica & 0 & 0 & 8 & 8 \\
Pruebas de chi-cuadrado & 1 & 4 & 30 & 35 \\
Chi-cuadrado de Pearson & Valor & & gl & Sig. asintótica (bilateral) \\
Razón de verosimilitudes & $1.728^{\mathrm{a}}$ & 2 & .421 \\
Asociación lineal por lineal & 2.833 & 2 & .243 \\
N de casos válidos & 1.486 & 1 & & .223
\end{tabular}

a. 4 casillas $(66.7 \%)$ tienen una frecuencia esperada inferior a 5. La frecuencia mínima esperada es .23.

Fuente: Elaboración propia

Los resultados obtenidos de la prueba del chi-cuadrado, permiten visualizar indudablemente que no existe una relación entre la variable de inclusión educativa y trabajo colaborativo, debido a que los docentes aplican el trabajo colaborativo de la forma tradicional y generalmente sin respetar el proceso necesario para una correcta ejecución del mismo, ello a pesar de que la mayoría está consciente que la inclusión educativa se enfoca en atender las necesidades educativas de los estudiantes; por lo que para solucionar estos inconvenientes, es importante la capacitación en cuanto a metodologías 
CIENCIAMATRIA

Revista Interdisciplinaria de Humanidades, Educación, Ciencia y Tecnología

Año VI. Vol. VI. N¹. Edición Especial. 2020

Hecho el depósito de ley: pp201602FA4721

ISSN-L: 2542-3029; ISSN: 2610-802X

Universidad Nacional Experimental Francisco de Miranda (UNEFM). Santa Ana de Coro. Venezuela

Marcia Lorena Cabrera-Abad; Darwin Gabriel García-Herrera; María Isabel Álvarez-Lozano; Juan Carlos Erazo-Álvarez

activas, como el trabajo colaborativo para permitir la participación de los estudiantes de inclusión en las aulas.

Tabla 2

Inclusión es solo la inserción de niños a planteles regulares

\begin{tabular}{cccccc} 
& Frecuencia & Porcentaje & $\begin{array}{c}\text { Porcentaje } \\
\text { válido }\end{array}$ & $\begin{array}{c}\text { Porcentaje } \\
\text { acumulado }\end{array}$ \\
\hline \multirow{4}{*}{ Válidos } & $\begin{array}{c}\text { Completamente en } \\
\text { desacuerdo }\end{array}$ & 1 & 2.9 & 2.9 & 2.9 \\
& $\begin{array}{c}\text { Algo de acuerdo } \\
\text { Completamente de acuerdo }\end{array}$ & 8 & 22.9 & 22.9 & 25.7 \\
& Total & 35 & 74.3 & 74.3 & 100.0 \\
& 100.0 & 100.0 & \\
\hline
\end{tabular}

Fuente: Elaboración propia

De la encuesta desarrollada en lo referente a la variable de inserción de niños a planteles regulares, los docentes consideran que con la admisión de todos los niños, sin distinción, a las instituciones fiscales, particulares y fiscomisionales; es suficiente para que formen parte de una sociedad inclusiva, cuando es precisamente allí donde por lo general empieza la exclusión, por parte de la comunidad educativa hacia los estudiantes con necesidades educativas especiales, al prestar poco interés en aquellos que más lo necesitan, por falta de mayor sensibilización y capacitación de los actores de proceso educacional.

\section{Tabla 3}

Utilización de metodologías activas para estudiantes NEE

Frecuencia Porcentaje Porcentaje válido Porcentaje acumulado

\begin{tabular}{cccccc}
\hline \multirow{3}{*}{ Válidos } & A veces & 18 & 51.4 & 51.4 & 51.4 \\
& Siempre & 17 & 48.6 & 48.6 & 100.0 \\
& Total & 35 & 100.0 & 100.0 & \\
\hline
\end{tabular}

Fuente: Elaboración propia 


\section{CIENCIAMATRIA}

Revista Interdisciplinaria de Humanidades, Educación, Ciencia y Tecnología

Año VI. Vol. VI. N¹. Edición Especial. 2020

Hecho el depósito de ley: pp201602FA4721

ISSN-L: 2542-3029; ISSN: 2610-802X

Universidad Nacional Experimental Francisco de Miranda (UNEFM). Santa Ana de Coro. Venezuela

Marcia Lorena Cabrera-Abad; Darwin Gabriel García-Herrera; María Isabel Álvarez-Lozano; Juan Carlos Erazo-Álvarez

Esta tabla nos muestra la realidad de muchas de las instituciones del país, en donde muy pocas veces se aplican metodologías activas en el aula y sobre todo tomando en cuenta que la participación de los estudiantes con NEE sea proporcional a la de los demás estudiantes.

\section{Tabla 4}

Búsqueda de estrategias para estudiantes con NEE

\begin{tabular}{cccccc} 
& & Frecuencia & Porcentaje & Porcentaje válido & $\begin{array}{c}\text { Porcentaje } \\
\text { acumulado }\end{array}$ \\
\hline \multirow{4}{*}{ Válidos } & Nunca & 1 & 2.9 & 2.9 & 2.9 \\
& A veces & 13 & 37.1 & 37.1 & 40.0 \\
& Siempre & 21 & 60.0 & 60.0 & 100.0 \\
& Total & 35 & 100.0 & 100.0 & \\
\hline
\end{tabular}

Fuente: Elaboración propia

La información de este apartado nos deja clara la contradicción de opinión de los docentes, con relación a la tabla anterior ya que solo a veces aplican metodologías para estudiantes con NEE, pero siempre buscan estrategias para los mismos, situación que es muy confusa ya que si no tenemos una metodología que proporcione estrategias, éstas solas no tendrían sentido.

\section{Tabla 5}

Grupo focal sobre inclusión educativa y trabajo colaborativo

\begin{tabular}{|c|c|c|}
\hline $\begin{array}{l}\text { Unidad de } \\
\text { análisis }\end{array}$ & Categoría & Segmento \\
\hline Inclusión & Conocomiento & - Todos los niños que son discriminados por diferentes motivos, \\
\hline \multirow[t]{4}{*}{ Educativa } & de los & si se hace una discrimanción a esos niños se esta ejecutando \\
\hline & docentes sobre & una inclusión. \\
\hline & inclusión & \\
\hline & educativa & \\
\hline
\end{tabular}




\section{CIENCIAMATRIA}

Revista Interdisciplinaria de Humanidades, Educación, Ciencia y Tecnología

Año VI. Vol. VI. N¹. Edición Especial. 2020

Hecho el depósito de ley: pp201602FA4721

ISSN-L: 2542-3029; ISSN: 2610-802X

Universidad Nacional Experimental Francisco de Miranda (UNEFM). Santa Ana de Coro. Venezuela

Marcia Lorena Cabrera-Abad; Darwin Gabriel García-Herrera; María Isabel Álvarez-Lozano; Juan Carlos Erazo-Álvarez

- La inclusión viene de la exclusión que busca un derecho a la educación de calidad y por lo tanto no se debe menospreciar a ningún niño.

- Un niño excluido ya debe considerarse como estudiantes con NEE, además es el trato igualitario para todos los estudiantes y que se sientan bien en el aula.

- No debe importar que sean pobres, ricos, inteligentes o no, cultura, religión, etc. A veces depende de los problemas del hogar y hay que atender esa diversidad.

$\begin{array}{ll}\text { Aprendizaje } & \text { Utilización de } \\ \text { de } & \text { estrategias } \\ \text { estudiantes } & \text { para trabajar } \\ \text { con } & \text { con } \\ \text { necesidades } & \text { estudiantes } \\ \text { educativas } & \text { NEE } \\ \text { especiales } & \end{array}$

- Los docentes no buscamos estrategias para ayudar a los estudiantes con NEE, cuando observamos que no aprende lo enviamos al Departamento de Consejería Estudiantil [DECE] y esperamos que ellos lo solucionen, deberíamos trabajar en eso pero no lo hacemos.

- Yo he observado que ha habido un cambio con relación a años atrás nadie quería reconocer que en la Unidad Educativa habían niños con NEE y ahora ya por lo menos se reconoce. Sin embargo sigue existiendo el hecho de que los docentes no buscamos la metodología para ayudar a esos estudiantes, a esos niños por lo general se les pone a dibujar o graficar.

- Es necesaria la participacion del DECE por que ellos son los que diagnostican y ayudan a estos estudiantes. Yo no estoy preparada dentro de alguna rama de la psicología para atender en su totalidad a los estudiantes con NEE.

- Empezando desde las grandes organizaciones como distritos, UDAI, Autoridades, Instituciones Educativas; se debería hacer un cambio y trabajar en conjunto por estos estudiantes. Si esperamos del DECE a veces se demora y hay tiempo perdido sin trabajar como se debería con los niños de inclusión, a veces hay que seguir el instinto y ademas deberíamos auto educarnos para ayudar a estos estudiantes porque no tenemos los conocimientos necesarios y por lo tanto no lo aplicamos. 


\section{CIENCIAMATRIA}

Revista Interdisciplinaria de Humanidades, Educación, Ciencia y Tecnología

Año VI. Vol. VI. N¹. Edición Especial. 2020

Hecho el depósito de ley: pp201602FA4721

ISSN-L: 2542-3029; ISSN: 2610-802X

Universidad Nacional Experimental Francisco de Miranda (UNEFM). Santa Ana de Coro. Venezuela

Marcia Lorena Cabrera-Abad; Darwin Gabriel García-Herrera; María Isabel Álvarez-Lozano; Juan Carlos Erazo-Álvarez

$\begin{array}{ll}\text { Trabajo } & \text { Aplicación } \\ \text { colaborativo } & \text { correcta del } \\ & \text { Trabajo } \\ & \text { colaborativo }\end{array}$

- Busco estrategias para formar los grupos con juegos, fichas, etc. Los niños de inclusión siempre tienen un compañero tutor, se les motiva a todos los estudiantes para que les ayuden. Pero el trabajo colaborativo se utiliza a veces para tener tiempo de hacer planificaciones o algún trabajo que tiene el docente y deja que realicen el trabajo los estudiantes solos, para que luego sustenten. Los trabajos grupales se estan mal interpretando e incluso son temidos por los estudiantes ya que la nota es para todo el grupo.

- Con mis niños hago tarjetas de colores y les hago escoger para formar los grupos y se trabaja el tema. En el trabajo colaborativo puede haber docentes que usen el tiempo del trabajo colaorativo para hacer otras actividades, en mi caso no se da porque siempre estoy pendiente de lo que están haciendo.

- En los grupos trato de insentivar los valores como compartir debido a que son niños pequeños. En cuanto al trabajo colaborativo los docentes deberiamos tomar conciencia de como se debe hacer un trabajo grupal, no como lo hemos venido ejecutando sino planificar y no que sea algo improvisado.

- Les doy una tarea al grupo tratando de incluir a los estudiantes con NEE tratando de ver sus potencialidades para que colabore. El trabajo colaborativo se aplica como nos enseñaron a nosotros debido a que no asistimos a capacitaciones y sin duda va cambiando pero no tomamos conciencia en mejorar y realizar correctamente las metodologías.

Fuente: Elaboración propia

Después de haber hecho un análisis tanto de la encuesta, como el grupo focal realizado a los docentes de la Unidad Educativa Los Cañaris, se puede determinar que, sin duda existe una conciencia y aceptación sobre la inclusión educativa, sin embargo es importante reforzar ese arduo trabajo que realizan los docentes en las aulas, sobre todo 


\section{CIENCIAMATRIA}

Revista Interdisciplinaria de Humanidades, Educación, Ciencia y Tecnología

Año VI. Vol. VI. N¹. Edición Especial. 2020

Hecho el depósito de ley: pp201602FA4721

ISSN-L: 2542-3029; ISSN: 2610-802X

Universidad Nacional Experimental Francisco de Miranda (UNEFM). Santa Ana de Coro. Venezuela

Marcia Lorena Cabrera-Abad; Darwin Gabriel García-Herrera; María Isabel Álvarez-Lozano; Juan Carlos Erazo-Álvarez

con los estudiantes con necesidades educativas especiales, que no reciben las atenciones y la preocupacion necesaria para que su desarrollo en al ámbito escolar sea el apropiado, tanto intelectual como psicológicamente.

Cabe recalcar que el docente no es el responsable directo de lo que ha sucedido en los ultimos años ya que la mayoría venía de una educación tradicionalista, no es tan fácil cambiar esas ideas, el docente actual debe estar a la par con la tecnología, con las nuevas metodologías y con las exigencias que la sociedad hoy reclama, para brindar una educación de calidad teniendo en cuenta las diferencias de cada uno de sus estudiantes. En este sentido, (Navarro-Montaño, 2017), realizó un estudio en Venezuela en donde refiere que es indispensable que se mejore la educación, que se busque las estrategias, para obtener la tan anhelada educación de calidad, ya que la inclusión en muchos de los casos se entiende como integración, para esto se debe empezar por la aceptación de la diversidad en las aulas e incluso con toda la comunidad educativa, dentro de un estadio educativo con cero discriminaciones.

Según esta investigación se ha encontrado que una de las primeras deficiencias, es la falta de planificación, materiales y recursos que coadyuven a esta misión, con el aporte de cada uno de los actores de la educación, lo que concuerda con los resultados de la presente investigación ya que en este caso también se requiere sobre todo de un refuerzo en las metodologías que utiliza el docente en las aulas.

Se debe tener en cuenta también el aporte de (Ramírez, 2017) en su estudio realizado en Colombia, donde comenta que de acuerdo con los resultados obtenidos la inclusión debe ser vista como una oportunidad de los niños, niñas y jóvenes para ser más libres, motivados a alcanzar sus metas. En cuanto a la percepción, manifiesta que el docente está siempre buscando diferentes formas de incluir a todos los estudiantes en su proceso de distintas formas aunque posiblemente no sean las adecuadas.

En cambio en el caso de la familia, todavía no está consciente de las necesidades de estos niños, solo han dejado este trabajo en manos del docente quien en muchas ocasiones se ha convertido en alguien demasiado permisivo para con estos estudiantes, buscando darle el espacio que necesita, que también podría ser perjudicial, por lo que 
CIENCIAMATRIA

Revista Interdisciplinaria de Humanidades, Educación, Ciencia y Tecnología

Año VI. Vol. VI. N¹. Edición Especial. 2020

Hecho el depósito de ley: pp201602FA4721

ISSN-L: 2542-3029; ISSN: 2610-802X

Universidad Nacional Experimental Francisco de Miranda (UNEFM). Santa Ana de Coro. Venezuela

Marcia Lorena Cabrera-Abad; Darwin Gabriel García-Herrera; María Isabel Álvarez-Lozano; Juan Carlos Erazo-Álvarez

solicitan tener la posibilidad de contar con el apoyo de un profesional en inclusión para mejorar el desarrollo de las clases lo que se relaciona a los resultados encontrados sobre todo en el grupo focal donde los docentes manifiestan que permiten que los estudiantes con necesidades educativas especiales realicen cualquier actividad, como dibujar o realizar gráficos sin darse cuenta de lo perjudicial que resulta para el educando.

\section{PROPUESTA}

En los docentes recae generalmente el mayor peso de la responsabilidad sobre el futuro de la niñez y adolescencia que pasan por sus aulas y en ese futuro se verá reflejada la enseñanza de los maestros que a lo largo de su vida formaron parte de su educación, es por eso que el proceso enseñanza-aprendizaje va mucho más allá de ser el guía de los conocimientos que imparten en el aula de clases, por cuanto debe tener en cuenta varios otros aspectos relacionados indirectamente con la ardua labor educativa. Dicho lo anterior esta propuesta se enfoca especialmente en los estudiantes con Necesidades Educativas Especiales y entiéndase a éstos no solo a aquellos que tienen una discapacidad intelectual o física, sino también a educandos con vulnerabilidad por distintas razones, como violencia intrafamiliar, abuso sexual, trastornos psicológicos y otros.

En consecuencia aplicado correctamente este proceso, será beneficioso incluso para los demás estudiantes, a quienes se les hace partícipes del actuar educativo durante la mayor parte del tiempo, lo que redunda incluso en la comunidad educativa, ya que los docentes tendrán a su alcance una metodología que les permita manejar los tiempos que tanta falta hace, sacando a relucir las habilidades, potencialidades y destrezas de sus dirigidos; por otro lado fomentará valores que hoy en día se han visto afectados como el respeto, la responsabilidad, la solidaridad, el compañerismo, entre otros, con lo cual, sin duda padres y representantes legales, estarán extasiados con el avance de aquellos niños a los que les habían perdido la esperanza. 
CIENCIAMATRIA

Revista Interdisciplinaria de Humanidades, Educación, Ciencia y Tecnología

Año VI. Vol. VI. N¹. Edición Especial. 2020

Hecho el depósito de ley: pp201602FA4721

ISSN-L: 2542-3029; ISSN: 2610-802X

Universidad Nacional Experimental Francisco de Miranda (UNEFM). Santa Ana de Coro. Venezuela

Marcia Lorena Cabrera-Abad; Darwin Gabriel García-Herrera; María Isabel Álvarez-Lozano; Juan Carlos Erazo-Álvarez

El trabajo colaborativo bien ejecutado permitirá que los niños antes excluidos sean una pieza fundamental, en el cumplimiento de las metas que el docente proponga y para esto se debe considerar el siguiente proceso:

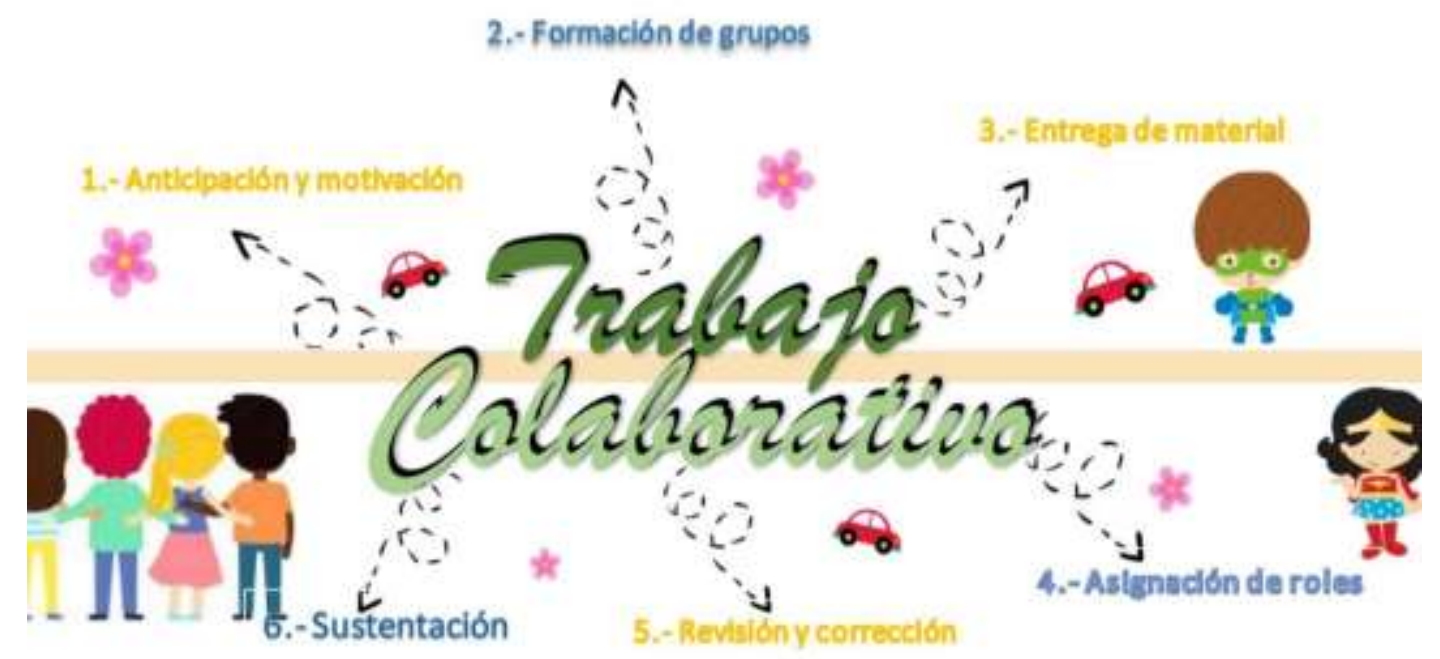

Figura 1. Propuesta para trabajar con estudiantes de inclusión. Fuente: Elaboración propia

A continuación, se detalla el proceso reflejado en la figura 1:

1. Anticipación y motivación: Es importante que antes de empezar a trabajar los temas en los grupos, se les sugestione por medio de videos, imágenes, material lúdico o información que el docente considere, para introducir a los estudiantes en los nuevos temas y tengan una idea de lo que se va a trabajar posteriormente, permitiendo que su creatividad vaya más allá del límite del aula. Además, no se debe olvidar que la motivación es un punto importante para buscar el interés de los educandos, esta se puede dar al inicio, durante y/o después de culminado el compromiso.

2. Formación de grupos: Para la formación de grupos, se pueden utilizar diferentes estrategias didácticas, en este apartado se debe tener en cuenta que cada estudiante de inclusión se encuentre en diferentes grupos con un estudiante guía, quien deberá ser sensibilizado a menudo por el docente para que la ayuda sea la 
adecuada y el estudiante con necesidades educativas especiales sea tratado de la misma forma que los demás sin discriminar.

3. Entrega de material: El o la docente debe preparar anticipadamente el material con el que van a trabajar los estudiantes dependiendo de los temas a tratar, este material debe ser comprensible de acuerdo al nivel en el que se trabaje, además se pueden entregar imágenes, fichas, material para maquetas, entre otros. Es importante que dentro de los grupos se fomente valores que beneficien a todos, especialmente a aquellos que siempre han sido discriminados, para lo cual se puede entregar una tarjeta amarilla y una roja, en caso de que alguien del grupo haga o diga algo indebido que afecte a otros estudiantes, obtendrá una tarjeta amarilla como primer llamado de atención y si el mismo estudiante vuelve a cometer un acto parecido, obtendrá una tarjeta roja y tendrá que pedir una disculpa en frente de todos los estudiantes explicando cómo se sentiría si le hicieran lo mismo. La entrega de material queda a la imaginación del docente con el objetivo de que la meta que propone se cumpla a cabalidad y los estudiantes se sientan satisfechos con lo realizado.

4. Asignación de roles: En este apartado empecemos por el rol del docente, él o ella será un guía que se encuentre constantemente en cada uno de los grupos aportando con ideas y resolviendo dudas, es alguien activo que debe estar motivando constantemente para que logren llegar a la meta. Por otro lado, cada estudiante del grupo debe tener un rol importante o una tarea específica a realizar y si no cumpliera, el trabajo no podría culminar, es decir deben trabajar en equipo; en cuanto a los estudiantes con necesidades educativas especiales deben tener un rol de acuerdo a sus habilidades y quien mejor que el docente para conocerlas, rol o habilidad que debe ser muy importante, de tal suerte que su participación en el grupo sea una pieza clave.

5. Revisión y corrección: Los estudiantes harán una planificación de cómo elaborar el trabajo y presentarán un primer borrador al docente, que con aportes de los mismos integrantes del grupo por medio de preguntas exploradoras sobre lo 


\section{CIENCIAMATRIA}

Revista Interdisciplinaria de Humanidades, Educación, Ciencia y Tecnología

Año VI. Vol. VI. N¹. Edición Especial. 2020

Hecho el depósito de ley: pp201602FA4721

ISSN-L: 2542-3029; ISSN: 2610-802X

Universidad Nacional Experimental Francisco de Miranda (UNEFM). Santa Ana de Coro. Venezuela

Marcia Lorena Cabrera-Abad; Darwin Gabriel García-Herrera; María Isabel Álvarez-Lozano; Juan Carlos Erazo-Álvarez

elaborado se corrijan los errores y se realice el trabajo final, el docente no debe rechazar ningún punto de vista o idea de los que conforman el grupo, sino debe buscar la manera de hacerlos reflexionar en caso de que no esté claro el contenido.

6. Sustentación: Por lo general la sustentación es la parte más difícil para los estudiantes y sobre todo para los de inclusión, es por eso que en este apartado el docente debe incentivar a los estudiantes que se encuentran en el público para alentar a sus compañeros. Luego de haber generado un ambiente de confianza, cada estudiante debe sustentar una parte del tema compartiendo los comentarios y la forma como se trabajó en el grupo, no debe ser algo mecánico o estudiado, debe ser lo que entendió mientras elaboraban el trabajo, los demás integrantes pueden apoyar al sustentante cuando lo vean con dificultad. Es importante que, en el caso de los estudiantes con necesidades educativas especiales, la sustentación sea de la misma forma que su participación en el grupo, de acuerdo a sus potencialidades. En fin, la sustentación servirá para comentar la experiencia vivida dentro del grupo.

\section{CONCLUSIONES}

Lo importante de este trabajo investigativo, ha sido sin duda enfatizar en el tema de inclusión educativa, buscando la forma de que los estudiantes se sientan confiados en al ámbito educativo y que se sientan capaces de realizar todas las actividades de la misma forma que los demás, inclusión que debe estar en directa relación con el trabajo colaborativo, que es una metodología que si bien se usa en las aulas, muchas veces ésta no se lo hace de forma correcta, generalmente no se consideran como se debe a los estudiantes con necesidades educativas especiales, que es en quienes convergen las necesidades inherentes al cumplimiento de sus metas.

La participación que permite esta metodología es imprescindible, ya que es ahí donde existe un apoyo mutuo, no solo entre los integrantes del grupo, sino una participación directa y constante del docente que, considerando la diversidad de sus educandos en el 
CIENCIAMATRIA

Revista Interdisciplinaria de Humanidades, Educación, Ciencia y Tecnología

Año VI. Vol. VI. N¹. Edición Especial. 2020

Hecho el depósito de ley: pp201602FA4721

ISSN-L: 2542-3029; ISSN: 2610-802X

Universidad Nacional Experimental Francisco de Miranda (UNEFM). Santa Ana de Coro. Venezuela

Marcia Lorena Cabrera-Abad; Darwin Gabriel García-Herrera; María Isabel Álvarez-Lozano; Juan Carlos Erazo-Álvarez

aula, fomenta un espacio de superación y ganas de aprender, obteniendo resultados que antes no se veían reflejados, por falta de tiempo o metodologías que promuevan el respeto y apoyo al alumnado sin distinciones.

Es importante entonces el compromiso inmediato de los docentes a cambiar y mejorar el estilo de vida de los niños, niñas y adolescentes, que tienen razones suficientes para que su aprendizaje se vea afectado, ya sea por una discapacidad, por una situación de vulnerabilidad o por algún trastorno psicológico o del aprendizaje; teniendo en cuenta las repercusiones que representa mantener el actual programa educativo, lo cual hace que los estudiantes con necesidades educativas especiales no puedan actuar como el resto de compañeros en el aula, enfrentándose a escenarios excluyentes muchos ámbitos .

Es importante entonces el compromiso inmediato de los docentes a cambiar y mejorar el estilo de vida de los niños, niñas y adolescentes, que tienen razones suficientes para que su aprendizaje se vea afectado, ya sea por una discapacidad, por una situación de vulnerabilidad o por algún trastorno psicológico o del aprendizaje; teniendo en cuenta las repercusiones que representa mantener el actual programa educativo, lo cual hace que los estudiantes con necesidades educativas especiales no puedan actuar como el resto de compañeros en el aula, enfrentándose a escenarios excluyentes en todos los ámbitos, situación que sin duda debe cambiar para su beneficio.

\section{REFERENCIAS CONSULTADAS}

Aguilar, M. (2013). Educación, diversidad e inclusión: la educación intercultural en perspectiva [Education, diversity and inclusion: intercultural education in perspective]. Ra Ximhai, 9(1), 49-59. Obtenido de https://n9.cl/v4ohe

Álvarez, J., Grau, S., \& Pertegal, M. (2016). El trabajo colaborativo y la pedagogía del contexto. Innovación metodológica [Collaborative work and pedagogy of context. Methodological innovation]. Opción, 32(7), 74-96. Obtenido de https://n9.cl/ro01

Argudo-Tello, K. J., Erazo-Álvarez, J. C., \& Narváez-Zurita, C. I. (2019). Evaluación de Control Interno en Riesgos Estratégicos para la Dirección de Planificación de la Universidad de Cuenca. [Evaluation of Internal Control in Strategic Risks for the 


\section{CIENCIAMATRIA}

Revista Interdisciplinaria de Humanidades, Educación, Ciencia y Tecnología

Año VI. Vol. VI. N¹. Edición Especial. 2020

Hecho el depósito de ley: pp201602FA4721

ISSN-L: 2542-3029; ISSN: 2610-802X

Universidad Nacional Experimental Francisco de Miranda (UNEFM). Santa Ana de Coro. Venezuela

Marcia Lorena Cabrera-Abad; Darwin Gabriel García-Herrera; María Isabel Álvarez-Lozano; Juan Carlos Erazo-Álvarez

Planning Direction of the University of Cuenca]. Revista Arbitrada Interdisciplinaria Koinonía, 67-96. doi:http://dx.doi.org/10.35381/r.k.v4i1.372

Ávila-Sacoto, E., Erazo-Álvarez, J. C., Narváez-Zurita, C. I., \& Erazo-Álvarez, C. A. (2019). Estrategias de marketing digital 2.0 para la generación de ingresos en Pymes de servicios. [Digital Marketing 2.0 strategies for generating income in service Pymes]. Cienciamatria, 187-214. doi:https://doi.org/10.35381/cm.v5i1.264

Arroyo-Carrera, E., Loor-Santos, M., Mendoza-Mera, J., \& Solorzano-Zambrano, M. (2020). Gestión de aprendizaje creativo mediante la Herramienta Powtoon en estudiantes de lengua y literatura. [Creative learning management through the Powtoon Tool in language and literature students]. EPISTEME KOINONIA, 3(5), 251-267. http://dx.doi.org/10.35381/e.k.v3i5.775

Argandoña-Mendoza, M., García-Mejía, R., Ayón-Parrales, E., \& Zambrano-Zambrano, Y. (2020). Investigación e innovación educativa: Reto escolar por COVID-19 en el Ecuador. KPISTEME KOINONIA, 3(5), 162-182. http://dx.doi.org/10.35381/e.k.v3i5.726

Calle-Abril, D. R., Erazo-Álvarez, J. C., \& Narváez-Zurita, C. I. (2020). Estrategias de mediación y solución de conflictos para el sector industrial de pinturas [Mediation and conflict resolution strategies for the paint industry]. Revista Arbitrada Interdisciplinaria Koinonía, 514-544. doi:http://dx.doi.org/10.35381/r.k.v5i10.704

Dabdub-Moreira, M., \& Pineda-Cordero, A. (2015). La atención de las necesidades educativas especiales y la labor docente en la escuela [The attention of the special educational needs and the educational work in the school]. Revista Costarricense de Psicología, 34(1), 41-55. Obtenido de https://n9.cl/z564

Domínguez, J., López, A., Pino, M., \& Vázquez, E. (2015). Integración o inclusión: El dilema educativo en la atención a la diversidad [Integration or inclusion: The educational dilemma in attention to diversity]. Revista Portuguesa de Educação, 28(2), 31-50. Obtenido de https://n9.cl//364

Echeita, G., \& Duk, C. (2008). Inclusión educativa [Educational inclusion]. Red Iberoamericana de Investigación Sobre Cambio y Eficacia Escolar, 1-8. Obtenido de https://n9.cl/g62j

Fernández, J. (2013). Competencias docentes y educación inclusiva [Teaching skills and inclusive education]. REDIE. Revista Electrónica de Investigación Educativa, 15(2), 82-99. Obtenido de https://n9.cl/gdlio 


\section{CIENCIAMATRIA}

Revista Interdisciplinaria de Humanidades, Educación, Ciencia y Tecnología

Año VI. Vol. VI. N¹. Edición Especial. 2020

Hecho el depósito de ley: pp201602FA4721

ISSN-L: 2542-3029; ISSN: 2610-802X

Universidad Nacional Experimental Francisco de Miranda (UNEFM). Santa Ana de Coro. Venezuela

Marcia Lorena Cabrera-Abad; Darwin Gabriel García-Herrera; María Isabel Álvarez-Lozano; Juan Carlos Erazo-Álvarez

García-Cedeño, G., Vélez-Loor, M., Franco-Zambrano, C., \& Ormaza-Bermello, M. (2020). Educación por competencias: Un reordenamiento curricular durante emergencia escolar por COVID-19. [Competency Education: A Curricular Rearrangement During School Emergency by COVID-19]. EPISTEME KOINONIA, 3(5), 221-235. http://dx.doi.org/10.35381/e.k.v3i5.770

González-Vargas, J. (2014). Una mirada del trabajo colaborativo en la escuela primaria desde las representaciones sociales [A look at collaborative work in primary school from social representations]. Ra Ximhai, 10(5), 115-134. Obtenido de https://n9.cl/ai29

Hamui-Sutton, A., \& Varela-Ruiz, M. (2013). La técnica de grupos focales [The focus group technique]. Investigación en Educación Médica, 2(5), 55-60. Obtenido de https://n9.cl/5gix

Martín, D., González, M., Navarro, Y., \& Latingua, L. (2017). Teorías que promueven la inclusión educativa [Theories that promote educational inclusion]. Atenas, 1-7. Obtenido de https://n9.cl/xt8i

Navarro-Montaño, M. (2017). Mejorar la calidad de la educación inclusiva: propuesta de indicadores [Improving the quality of inclusive education: proposal of indicators]. Revista de Pedagogía, 38(102), 122-143. Obtenido de https://n9.cl/79xf

Opertti, R. (2009). La Educación Inclusiva, perspectiva internacional y retos de futuro [Inclusive Education, international perspective and future challenges]. Recuperado el 2020, de unesco.org: https://n9.cl/uhkmk

Peche-Cruz, H., \& Giraldo-Supo, V. (2019). El Aprendizaje Flip Learning centrado en el estudiante como generador de calidad educativa. [Student-centered Flip Learning as a generator of educational quality]. Revista Arbitrada Interdisciplinaria Koinonía, 4(8), 427-450. http://dx.doi.org/10.35381/r.k.v4i8.293

Quevedo-Álava, R., Corrales-Moreno, L., Palma-Delgado, G., \& Mendoza-Suárez, G. (2020). Psicopedagogía y TIC en período de COVID-19. Una reflexión para el aprendizaje significativo. [Psychopedagogy and ICT in the COVID-19 period. A Reflection for Meaningful Learning]. EPISTEME KOINONIA, 3(5), 202-220. http://dx.doi.org/10.35381/e.k.v3i5.769

Ramírez, W. (2017). La inclusión: una historia de exclusión en el proceso de enseñanzaaprendizaje [Inclusion: a history of exclusion in the teaching-learning process]. Cuadernos de Lingüística Hispánica(30), 211-230. Obtenido de https://n9.cl/b1qv 
CIENCIAMATRIA

Revista Interdisciplinaria de Humanidades, Educación, Ciencia y Tecnología

Año VI. Vol. VI. N¹. Edición Especial. 2020

Hecho el depósito de ley: pp201602FA4721

ISSN-L: 2542-3029; ISSN: 2610-802X

Universidad Nacional Experimental Francisco de Miranda (UNEFM). Santa Ana de Coro. Venezuela

Marcia Lorena Cabrera-Abad; Darwin Gabriel García-Herrera; María Isabel Álvarez-Lozano; Juan Carlos Erazo-Álvarez

Sarduy, Y. (2007). El análisis de información y las investigaciones cuantitativa y cualitativa [Information analysis and quantitative and qualitative research]. Revista Cubana de Salud Pública, 33(3). Obtenido de https://n9.cl/owkb

Saltos-Cedeño, A., Vallejo-Valdivieso, P., \& Moya-Martínez, M. (2020). Innovación en educación matemática de básica superior durante el confinamiento por COVID19. [Innovation in higher basic mathematics education during confinement by COVID-19]. EPISTEME KOINONIA, $\quad 3(5), \quad 142-161$. http://dx.doi.org/10.35381/e.k.v3i5.723

Vega, A. (2005). Integración de alumnas con necesidades educativas: ¿coherencia entre los discursos y las prácticas pedagógicas ejercidas por los profesores básicos? [Integration of students with educational needs]. Universidad de Chile. Obtenido de https://n9.cl/8jdu

Vélez-Loor, M., Vallejo-Valdivieso, P., \& Moya-Martínez, M. (2020). Recursos didácticos virtuales en proyectos de ciencias naturales en período de confinamiento por COVID-19. [Virtual teaching resources in natural science projects in confinement period by COVID-19]. EPISTEME KOINONIA, 3(5), 183-201. http://dx.doi.org/10.35381/e.k.v3i5.760

2020 por los autores. Este artículo es de acceso abierto y distribuido según los términos y condiciones de la licencia Creative Commons Atribución-NoComercial-Compartirlgual 4.0 Internacional (CC BY-NC-SA 4.0)

(https://creativecommons.org/licenses/by-nc-sa/4.0/). 